\title{
The Next Generation Textile Antennas Based on Substrate Integrated Waveguide Technology
}

\author{
Sam Agneessens ${ }^{*}$, Sam Lemey ${ }^{1}$, Riccardo Moro ${ }^{2}$, Maurizio Bozzi ${ }^{2}$, and Hendrik Rogier ${ }^{1}$ \\ ${ }^{1}$ Dept. of Information Technology (INTEC), IMEC-Ghent University, Sint-Pietersnieuwstraat 41, 9000 Ghent, Belgium, \\ email: sam.agneessens@intec.ugent.be,sam.lemey@intec.ugent.be, hendrik.rogier@intec.ugent.be
}

${ }^{2}$ Dep. of Electrical, Computer and Biomedical Engineering, University of Pavia, Via Ferrata, 1, 27100 Pavia, Italy, email: riccardo.moro@unipv.it,maurizio.bozzi@unipv.it

\begin{abstract}
Textile antennas for body-worn applications have some very specific requirements and needs. From an electrical engineer's point of view, good radiation characteristics and impedance matching to the active electronics are important. From the wearer's perspective, the antenna should be unobtrusively integrated into the clothing, and the smart textile comfortable to wear. New techniques offer the potential to fulfill these different needs. One new approach consists of applying metalized eyelets to implement substrate integrated waveguide technology on textile materials. This results in high-performance on-body antennas with excellent behavior in close proximity of the human body. Two realizations are discussed: a wideband design and a miniaturized half mode substrate integrated waveguide dual-band design. Both yield excellent free-space and on-body performance, and superb antenna-body isolation, automatically resulting in very robust characteristics when deployed on-body.
\end{abstract}

\section{Introduction}

In the last decade, developments in the domain of wearable textile electronic circuits have opened exciting new possibilities for smart fabric/integrated textile (SFIT) systems. Yet, up to now, the massive research efforts have not led to extensive market penetration of wearable electronic systems. The main impediments, which still must be resolved, concern robustness, cost, comfort and compactness of wearable electronic components. Therefore, in this contribution, the combination of substrate integrated waveguide techniques with textile antenna technology is adopted to leverage a new generation of compact and comfortable innovative wearable antenna designs that yield high performance, even in very close proximity of the human body.

Both in terms of impedance matching and radiation performance, a good on-body antenna requires robust characteristics, independent of the wearer's morphology or its position on the body. These characteristics can be achieved by designing the antenna in such a way that the radiation pattern is directed away from the body. The advantages of such an approach are threefold: First of all, the antenna characteristics will be stable, independent of the presence of a wearer, since the body is 'invisible' to the antenna. Second, power-efficient designs are obtained, as no power is lost due to unnecessary absorption by the human body. Related to this is the final advantage: the antenna is safe for the user, as he or she will not be exposed to the electromagnetic radiation being transmitted.

Cavity-backed slot antennas have the potential to fulfill these conditions, as they only receive and transmit power in one hemisphere and minimize the amount of radiation leakage in unwanted directions. These designs offer possibilities in terms of size reduction by using miniaturization techniques that rely on the symmetry of the field distribution in the cavity and can be implemented in textile materials by relying on substrate integrated waveguide (SIW) technology [1, 2 , $3,4]$.

\section{Benefits and Realization of SIW structures on Textile Materials}

SIW technology exhibits some features that are very attractive for smart textile applications. It enables the integration of components - both active and passive - into a single substrate by means of a low-cost production process and is proven to maintain its operability with conformal and flexible structures [5]. Undesired radiation is minimized by the rows of vias, which act as an effective electric wall. This greatly improves the isolation between human tissue and body-worn microwave devices, limiting (unwanted) body influence and making body absorption of EM radiation a non-issue, which is a large step forward compared to existing implementations for body-worn systems.

To achieve flexible, light and robust on-body devices, the materials from which they are assembled should be chosen with care. Both designs presented here are identical in terms of fabrication process and used materials. The antenna substrate is a closed cell expanded rubber, which is typically used as foam against impact in protective garments (e.g. firefighter suits). It possesses low moisture regain, good chemical stability, and good resistance against oils and solvents. The material exhibits a characterized $\varepsilon_{\mathrm{r}}$ of 1.475 and loss tangent, tan $\delta$, of 0.016 at $2.45 \mathrm{GHz}$. The conductive 
layers are realized with a copper-plated nylon tafetta electro-textile, with a sheet resistivity of $0.18 \mathrm{Ohm} / \mathrm{sq}$ at $2.45 \mathrm{GHz}$. The layers are attached to each other by means of a thermally-activated adhesive sheet.

SIW on textile substrates may be implemented by means of conductive thread [4] or by fixing metalized eyelets through the substrate materials $[1,2,3]$ to provide a good electrical contact between the conductive layers on each side of the substrate. The latter method is used for the designs presented here.

\section{SIW Antennas for On-Body Applications: Topologies and Design}

Two different antenna topologies, realized on textile materials, and relying on SIW technology for optimal on-body performance, are presented. The first is a wideband cavity backed slot antenna, which covers multiple frequency bands. The second is a dual-band half-mode SIW (HMSIW) design that is specifically developed to combine excellent on-body performance with small dimensions. We now discuss the operating principles and the design-specific details related to these two antennas.

\subsection{Wideband Cavity Backed Slot Antenna}

The wideband design [2], presented in Fig. 1, is based on a non-resonant slot, to separate an SIW cavity into two half-cavities, and a coplanar feed line, to inject power into the cavity. The dimensions of the cavity and the slot are carefully chosen in order to excite two hybrid mode combinations at different, neighboring frequencies. At the lowest frequency there is a weak $\mathrm{TE}_{110}$ and strong $\mathrm{TE}_{120}$ mode, out of phase with each other. The resulting electrical field will mainly be situated in the lower cavity. At the higher resonance frequency there is a combination of a strong $\mathrm{TE}_{110}$ and weak $\mathrm{TE}_{120}$ mode which are in phase, but due to the large magnitude difference there is a resulting dominant field located in the upper cavity. In both cases the large magnitude difference over the slot will cause it to radiate. The combination of two resonances near to each other yields a broadband wearable antenna, covering multiple adjacent frequency bands. The antenna considered here covers the $2.4 \mathrm{GHz}$ ISM band $([2.4-2.4835] \mathrm{GHz})$ and the LTE 7 band, both in uplink and downlink ([2.5-257] GHz and [2.62-2.69] GHz).

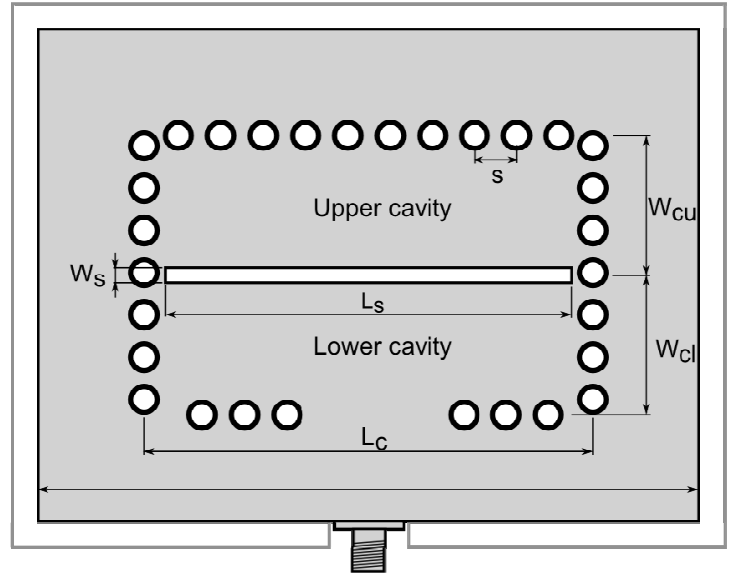

(a)

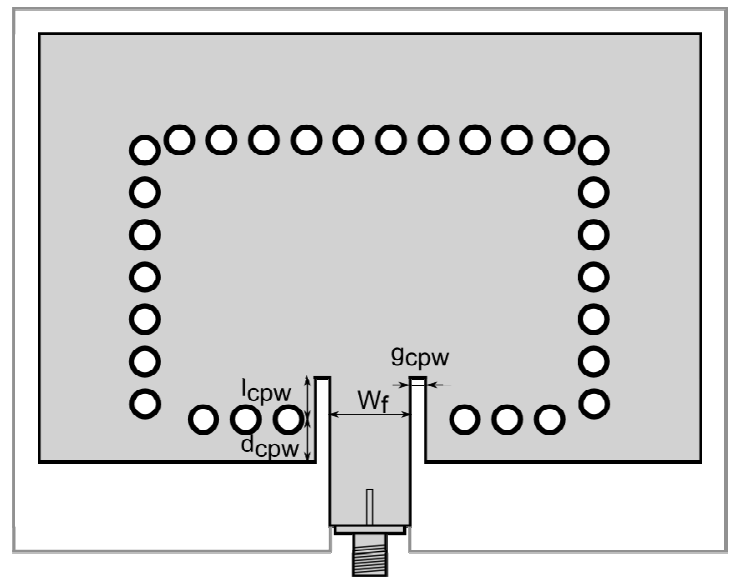

(b)

Fig. 1. Broadband antenna geometry (a) front view and (b) rear view. (Dimensions in $\mathrm{mm}$ : $\mathrm{L}_{\mathrm{c}}=84.5, \mathrm{~W}_{\mathrm{cu}}=26.2$, $\mathrm{W}_{\mathrm{cl}}=26.8, \mathrm{~W}_{\mathrm{s}}=3.9, \mathrm{~L}_{\mathrm{s}}=76.5, \mathrm{~W}_{\mathrm{f}}=15.5, \mathrm{~g}_{\mathrm{cpw}}=2.6, \mathrm{l}_{\mathrm{cpw}}=8, \mathrm{~d}_{\mathrm{cpw}}=7$ ).

\subsection{Compact Dual Band HMSIW Antenna}

The second design [3] also relies on the cavity-backed slot topology, now fed by a coaxial probe and with the slot along the diagonal of the cavity. Now, however, the focus is on miniaturization, to be able to achieve seamless integration into smart textiles. Two miniaturization techniques are implemented to reduce the size from the initial cavity-backed slot antenna to the final design. The HMSIW technique reduces the cavity size by half and an extra row of shorting vias reduces the dimensions again by almost $50 \%$. First, half of the cavity is removed, along the virtual magnetic wall. This results into an HMSIW antenna that operates in a single frequency band, with an upper and a lower cavity, separated by the slot. Further miniaturization is then achieved by adding a row of miniaturization vias above the slot, which effectively short circuit the upper cavity, leaving only the lower cavity. Dual-band operation is then achieved by adding a second slot. The design evolution and geometry are presented in Fig. 2.a and Fig. 2.b respectively.

Like the broadband design, this antenna also uses a ground plane to obtain excellent isolation between the body and the radiating antenna, with minimal radiation towards the wearer. The half diamond shaped HMSIW antenna is 
operated in dual band mode and is used to cover the $2.4 \mathrm{GHz}$ ISM band and the $5.8 \mathrm{GHz}$ ISM band, making it suitable for high data-rate communications relying on protocols such as IEEE 802.11.n (Wi-Fi), ZigBee, wireless HDMI etc.

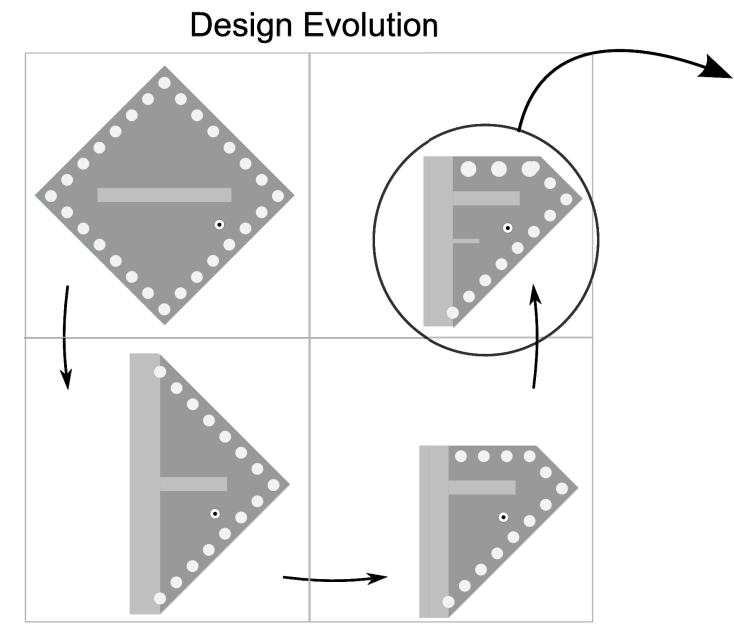

(a)

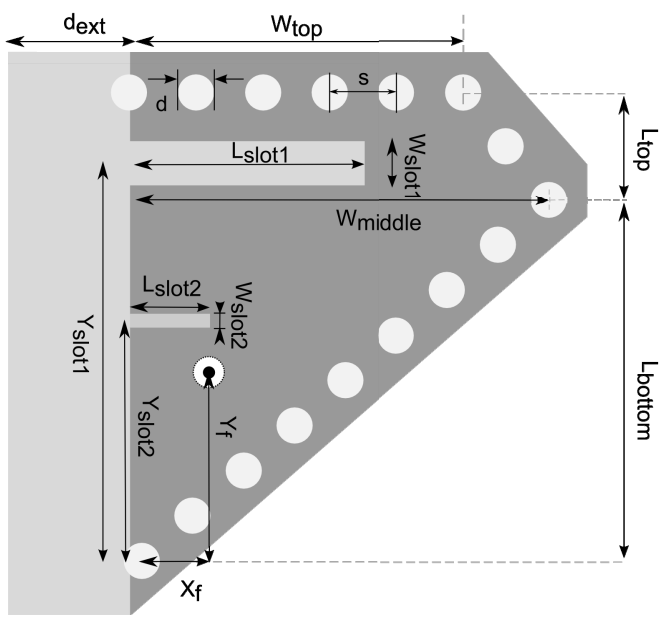

(b)

Fig. 2. Dual-band antenna (a) design evolution and (b) geometry. $\left(\mathrm{W}_{\text {middle }}=50.0, \mathrm{~L}_{\text {bottom }}=41.4, \mathrm{~L}_{\text {top }}=12.3, \mathrm{~W}_{\text {top }}=\right.$ $\left.39.7, \mathrm{~L}_{\text {slot } 1}=28.0, \mathrm{~W}_{\text {slot } 1}=4.9 \mathrm{~mm}, \mathrm{~L}_{\text {slpt2 }}=9.4, \mathrm{~W}_{\text {slot2 }}=1.5, \mathrm{X}_{\mathrm{f}}=20.0, \mathrm{Y}_{\mathrm{f}}=29.8, \mathrm{~d}_{\text {ext }}=14.6, \mathrm{~d}=4, \mathrm{~s}=8\right)$

\section{Performance in Free-Space}

This section discusses antenna performance in a free-space set up. After fabrication, as described in section II, the impedance matching and radiation pattern in a stand-alone situation are determined in an anechoic chamber, using an Agilent N5242A PNA-X Microwave Network Analyzer. These measurement results are compared to the simulated values.

As seen in Fig. 3.a the broadband antenna performs very well in free-space conditions. Good impedance matching to $\mathrm{Z}_{0}=50 \Omega$ from $2.356 \mathrm{GHz}$ to $2.74 \mathrm{GHz}$, results in a bandwidth of $409 \mathrm{MHz}$ or $15.1 \%$. The antenna completely covers the $2.4 \mathrm{GHz}$ ISM band and the up- and downlink frequency bands for 4G LTE 7 standard. The design goals are also met for the dual band antenna, for which the reflection coefficient is displayed in Fig. 3.b. The input return loss remains higher than $10 \mathrm{~dB}$ in the $2.4 \mathrm{GHz}$ and $5.8 \mathrm{GHz}$ ISM bands, with measured bandwidths of $4.9 \%$ and $5.1 \%$, in the low and high frequency band, respectively.

Free-space radiation patterns are simulated and measured for both antennas and, when compared, these values agree very well. The maximum simulated gain of the first antenna is $6.6 \mathrm{dBi}$ and the efficiency is $83 \%$ at the center frequency of the $2.4 \mathrm{GHz}$ ISM band, which compares well to the measured values of $4.7 \mathrm{dBi}$ gain and a radiation efficiency of $83 \%$. The dual-band half diamond shaped antenna boasts $4.2 \mathrm{dBi}$ and $5.5 \mathrm{dBi}$ simulated maximum gains, and simulated efficiencies equaling $71.1 \%$ and $74.0 \%$, in the upper and lower frequency range, respectively. Measurements also agree very well with gain values of $4.1 \mathrm{dBi}$ and $5.8 \mathrm{dBi}$, and $72.8 \%$ and $85.6 \%$ radiation efficiency in the lower and upper frequency band. Another important factor in antenna design for on-body deployment is the front-to-back ratio (FTBR), as this provides an indication of how good the body-antenna isolation will be. Both antennas perform well with a measured FTBR better than $16 \mathrm{~dB}$, for the broadband design, and better than $14.4 \mathrm{~dB}$ and $23.7 \mathrm{~dB}$, in the lower and upper frequency bands of the diamond shaped antenna.

\section{Performance in On-Body Deployment}

Since an on-body antenna will be used in smart textile applications, worn very close to the body, its performance and robustness should also be thoroughly tested to be certain that bending and body proximity do not cause unacceptable frequency detuning and/or loss of radiation performance. Therefore, suitable measurements to test these cases are conducted.

Both designs are stable in terms of frequency detuning and radiation performance when placed on the human body, as can be seen from Fig. 3. The reflection coefficient remains below $-10 \mathrm{~dB}$ in all of the frequency bands of interest. Loss of bandwidth is also minimized, with a bandwidth of $449 \mathrm{MHz}$ for the wideband design and $118 \mathrm{MHz}$ and $243 \mathrm{MHz}$ around the $2.45 \mathrm{GHz}$ and $5.8 \mathrm{GHz}$ center frequencies, for the dual-band antenna. Radiation patterns were also measured and the maximal gain of both antennas remains stable. The wideband design yields a maximal measured gain of $5.0 \mathrm{dBi}$. The values for the dual band antenna are $4.4 \mathrm{dBi}$ and $5.7 \mathrm{dBi}$, at $2.45 \mathrm{GHz}$ and $5.8 \mathrm{GHz}$, respectively. 


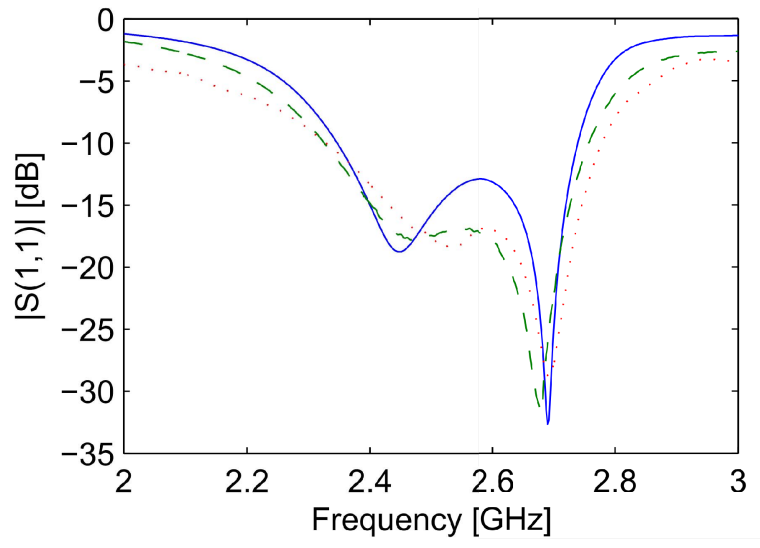

(a)

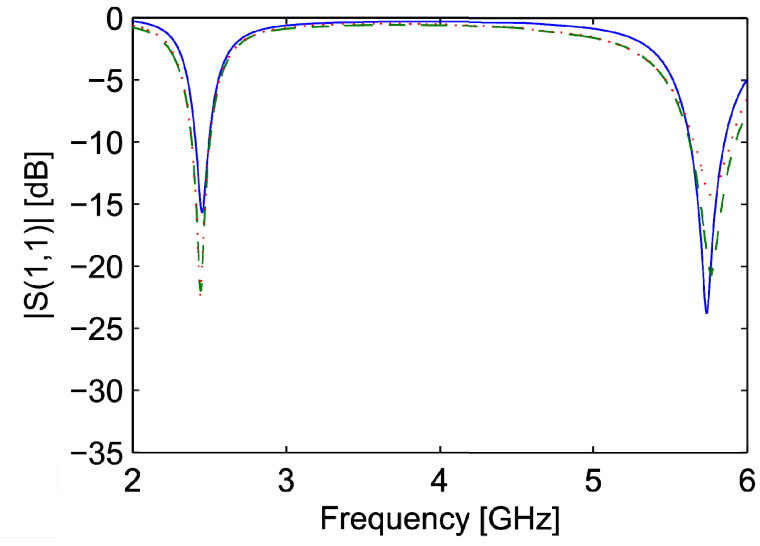

(b)

Fig. 3. Simulated (blue, full line), measured free-space (green, dashed line), and measured on-body (red, dotted line) reflection coefficient for the (a) wideband SIW antenna and (b) the dual-band HMSIW antenna.

These results compare very well to the free-space results, demonstrating that the influence of the body on the antenna performance remains minimal. This robust performance can be attributed to the use of a ground plane. This also leads to another major advantage of SIW cavity-backed slot topology for body worn applications: the antenna can be designed without having to include the body in the model, leading an easier and faster design process.

\section{Conclusions}

A new class of on-body antennas and their application techniques was presented. Two designs were discussed to show the merits and potential of this new design approach: a wideband SIW and a dual-band miniaturized HMSIW antenna. They rely on metalized eyelets to implement SIW technology on textile materials, which enables the development of conformal, light-weight antennas with high antenna-body isolation and robust performance, excellently suited for integration into smart textile systems. They boast excellent free-space performance and on-body performance, providing proof for the potential of SIW techniques for wearable antennas. The different layout and specs of the designs show the versatility of SIW techniques for deployment in diverse applications: large bandwidth, dual-band operation, and size miniaturization are achieved, while maintaining very robust on-body performance.

\section{References}

1. R. Moro, S. Agneessens, H. Rogier and M. Bozzi, "Wearable textile antenna in substrate integrated waveguide technology," Electronics letters, vol. 48, no. 16, pp. 985-987, 2012.

2. S. Lemey, F. Declercq and H. Rogier, "Dual-Band Substrate Integrated Waveguide Textile Antenna With Integrated Solar Harvester," Antennas and Wireless Propagation Letters, IEEE, vol. 13, pp. 269-272, 2014.

3. S. Agneessens and H. Rogier, "Compact Half Diamond Dual-Band Textile HMSIW On body Antenna," Antennas and Propagation, IEEE Transactions on, vol. 62, no. 99, p. in press, 2014.

4. T. Kaufmann and C. Fumeaux, "Wearable Textile Half-Mode Substrate-Integrated Cavity Antenna Using Embroidered Vias," Antennas and Wireless Propagation Letters, IEEE, vol. 12, pp. 805-808, 2013.

5. M. Bozzi, A. Georgiadis and K. Wu, "Review of substrate-integrated waveguide circuits and antennas," Microwaves, Antennas \& Propagation, IET, vol. 5, no. 8, pp. 909-920, 2011. 